\title{
A DUALIZING OBJECT APPROACH TO NONCOMMUTATIVE STONE DUALITY
}

\author{
GANNA KUDRYAVTSEVA
}

(Received 29 August 2011; accepted 1 March 2013; first published online 19 August 2013)

\author{
Communicated by M. Jackson
}

\begin{abstract}
The aim of the present paper is to extend the dualizing object approach to Stone duality to the noncommutative setting of skew Boolean algebras. This continues the study of noncommutative generalizations of different forms of Stone duality initiated in recent papers by Bauer and Cvetko-Vah, Lawson, Lawson and Lenz, Resende, and also the current author. In this paper we construct a series of dual adjunctions between the categories of left-handed skew Boolean algebras and Boolean spaces, the unital versions of which are induced by dualizing objects $\{0,1, \ldots, n+1\}, n \geq 0$. We describe the categories of Eilenberg-Moore algebras of the monads of the adjunctions and construct easily understood noncommutative reflections of left-handed skew Boolean algebras, where the latter can be faithfully embedded (if $n \geq 1$ ) in a canonical way. As an application, we answer the question that arose in a recent paper by Leech and Spinks to describe the left adjoint to their 'twisted product' functor $\omega$.
\end{abstract}

2010 Mathematics subject classification: primary 06E15; secondary 06E75, 18B30, 54B40.

Keywords and phrases: Boolean space, Boolean algebra, Stone duality, skew Boolean algebra, étale space, dualizing object, schizophrenic object, adjunction, monad, Eilenberg-Moore algebra, reflective subcategory.

\section{Introduction}

Stone duality [9, 14, 34] is probably the most famous result about Boolean algebras. It provides a subtle link between algebra and topology and has far-reaching consequences and numerous important generalizations.

Recently, different variations of Stone duality have been generalized to the noncommutative setting. We mention the generalizations to skew Boolean algebras by the author [16], to skew Boolean algebras with intersections independently by Bauer and Cvetko-Vah [2] and the author [16], to Boolean inverse semigroups by Lawson [20, 21], to some classes of pseudogroups by Lawson and Lenz [22], to pseudogroups and a class of quantales (at the level of objects) by Resende [31, 32]. Priestley duality

Author's research is partially supported by ARRS grant P1-0288.

(C) 2013 Australian Mathematical Publishing Association Inc. 1446-7887/2013 \$16.00 
has been generalized to the setting of skew lattices by Bauer, Cvetko-Vah, Gehrke, van Gool and the author in [3]. Very recently, Mark Lawson and the author have found a common generalization of the results of [16] and [20,21] to a duality for étale actions of Boolean inverse semigroups [19]. These actions were introduced in [13, 33] and play an important role in Morita theory of inverse semigroups [12, 33] as well as in the interplay of semigroup and topos theories [10, 11, 13, 18].

The study of skew Boolean algebras, the objects of consideration of this paper, was initiated by Cornish [8] and Leech [23, 24]. In [4] it was observed that skew Boolean algebras with intersections form a discriminator variety and therefore tools of universal algebra can be applied to their study. Stone duality for skew Boolean algebras [16] and skew Boolean algebras with intersections $[2,16]$ opens completely new perspectives of looking at these algebras. The duality of [16] made the constructions of this paper possible, and also, together with the results of [20, 21], led Mark Lawson and the author to the duality of [19]. The latter duality, in its turn, witnesses that skew Boolean algebras and Boolean inverse semigroups are closely related. Putting them together leads to the construction of new objects that are connected with important concepts of inverse semigroup theory and its applications.

A fundamental feature of Stone duality is that (the unital version of) it is induced by a dualizing object (sometimes also called a schizophrenic object) $\mathbf{2}=\{0,1\}$, considered as a Boolean algebra or as a discrete topological space. In contrast, its generalizations to the skew Boolean setting given in the papers $[2,16]$ do not possess this property (see Section 2.4 for details), despite the fact that there are natural candidates for the role of such objects: left-handed skew Boolean algebras $\mathbf{n}+\mathbf{2}=\{0,1, \ldots, n+1\}$. For $n=0$ such an algebra is just $\mathbf{2}$, and for $n \geq 0$ it generalizes $\mathbf{2}$ (see Subsection 2.2 for details). This suggests to study the constructions that are determined by these objects. In the present paper we treat this question and construct a series of dual adjunctions $\Lambda_{n} \dashv \lambda_{n}, n \geq 0$, between the categories of left-handed skew Boolean algebras and Boolean spaces, the unital versions of which are induced by the dualizing objects $\{0,1, \ldots, n+1\}, n \geq 0$. Our motivation comes from the well-established significance of dualizing objects, both from the universal algebra and the category theory viewpoints, (see, for example, [7, 15, 30]), on the one hand, and recently revealed importance of skew Boolean algebras, on the other hand.

Roughly speaking, our constructions are obtained if in the dualizing object approach to the classical Stone duality one replaces Boolean algebras by left-handed skew Boolean algebras, and $\mathbf{2}$ by $\mathbf{n}+\mathbf{2}, n \geq 0$ (but note that on the topological side we work with Boolean spaces, just as in the classical case). Therefore, the constructed adjunctions can be looked at as noncommutative generalizations of Stone duality. We emphasize that the constructions of this paper connect skew Boolean algebras with Boolean spaces and lead to dual adjunctions that are not equivalences, while the constructions of $[2,16]$ connect skew Boolean algebras or skew Boolean algebras with intersections with étale spaces over Boolean spaces and lead to dual equivalences.

It is worth noting that a variation of the functor $\lambda_{1}$ of this paper has previously appeared in another disguise in the paper by Leech and Spinks [27] as the 'twisted 
product' $\omega$-functor. Invoking Freyd's adjoint functor theorem, it was argued in [27] that the functor $\omega$ has a left adjoint, $\Omega$, and the question to describe $\Omega$ arose. As a first step towards answering this question, the action of $\Omega$ on finite objects was described in [27]. The approach of [27], however, did not allow to go further than that. In the present paper we look at both $\omega$ and $\Omega$ in a new light which brings more suitable insights and leads to the full description of the functor $\Omega$ (see Remarks 3.1 and 4.6).

We provide a nice combinatorial description of the monads induced by the constructed adjunctions and prove that the categories of Eilenberg-Moore algebras of these monads are equivalent to the images of $\lambda_{n}$, so that the adjunctions are monadic. Thus for each $n \geq 0$ the image of $\lambda_{n}$ is a reflective subcategory of the category of lefthanded skew Boolean algebras.

Given a left-handed skew Boolean algebra $S$, its maximal Boolean algebra quotient $S / \mathcal{D}$ reflects $S$ in the sense that there is a functor from the category of left-handed skew Boolean algebras to the category of Boolean algebras sending $S$ to $S / \mathcal{D}$, and this functor is the left adjoint to the inclusion functor going in the reverse direction. Thus $S / \mathcal{D}$ is known as a commutative reflection of $S$. In this paper we construct a series $\lambda_{n} \Lambda_{n}(S), n \geq 0$, of reflections of $S$. If $n=0$ we have $\lambda_{0} \Lambda_{0}(S) \simeq S / \mathcal{D}$. If $n \geq 1, \lambda_{n} \Lambda_{n}(S)$ is a noncommutative reflection of $S$. Moreover, the units of the constructed adjunctions provide a canonical way to faithfully represent $S$ in $\lambda_{n} \Lambda_{n}(S)$ (for all $n \geq 1$ ). However, in order to decrease the 'degree of noncommutativity' of the enveloping algebra one has to sacrifice the size of the underlying Boolean algebra $\left(\Lambda_{n}(S)\right)^{*}$. Note that the possibility of an embedding of $S$ into $\lambda_{n}(X)$ for some $X$ can be easily deduced from [27, Corollary 3.6] applying Remark 3.1 of this paper. But no specific construction of an embedding of a skew Boolean algebra into another skew Boolean algebra with 'low degree of noncommutativity' had been previously known.

Before stating in Sections 3-5 the main constructions and results of this paper, we collect in Section 2 all necessary preliminaries. In particular, we explain what precisely we mean by a dualizing object approach to the classical Stone duality, then provide necessary background on skew Boolean algebras and étale spaces and review the noncommutative Stone duality from [16] needed in this paper. We also explain why the latter duality and its specialization for skew Boolean algebras with intersections are not induced by a dualizing object.

\section{Preliminaries}

2.1. The dualizing object view of the classical Stone duality. By a Boolean algebra we mean what is usually called a generalized Boolean algebra, that is, a relatively complemented distributive lattice with a bottom element. A Boolean algebra with a top element will be called a unital Boolean algebra. A homomorphism $\varphi: B_{1} \rightarrow B_{2}$ of Boolean algebras is called proper [9], provided that for any $b \in B_{2}$ there exists $a \in B_{1}$ such that $\varphi(a) \geq b$. In this paper any homomorphism of Boolean algebras is assumed to be proper. By BA we denote the category of Boolean algebras and proper morphisms. 
By a Boolean space we mean what is usually called a locally compact Boolean space, that is, a Hausdorff space in which compact-open sets form a base of the topology. Note that compact Boolean spaces are usually referred to as Boolean spaces. By BS we denote the category of Boolean spaces and continuous proper maps (recall that a map of topological spaces is proper if inverse images of compact sets are compact sets).

Given a Boolean space $X$, all continuous maps $X \rightarrow\{0,1\}$, where $\{0,1\}$ is a discrete topological space, such that $f^{-1}(1)$ is a compact set, form a Boolean algebra denoted by $X^{*}$ and called the dual Boolean algebra of $X$. Note that the elements of $X^{*}$ can be looked at as proper maps from $X$ to the Sierpiński space $\{0,1\}$. The assignment $X \rightarrow X^{*}$ is the object part of the contravariant functor $\mathbf{A}: \mathrm{BS} \rightarrow \mathrm{BA}$. The restriction of $\mathbf{A}$ to the category of compact Boolean spaces is an enriched contravariant Homset functor, because given a compact Boolean space $X$, the unital Boolean algebra $X^{*}$ consists of all continuous maps $X \rightarrow\{0,1\}$ (note that such a map is automatically also proper).

The spectrum $B^{*}$ of a Boolean algebra $B$ is the set of all proper morphisms from $B$ to the two-element Boolean algebra $\mathbf{2}=\{0,1\}$. The set $B^{*}$ is equipped with the topology whose base is formed by the sets $M(a)=\left\{f \in B^{*}: f(a)=1\right\}, a \in B$. This space is a Boolean space and is called the dual space of $B$. It is well known that the topology on $B^{*}$ can be also characterized as the subspace topology of the product space $\{0,1\}^{B}$. The assignment $B \rightarrow B^{*}$ is the object part of the contravariant functor $\mathbf{S}: \mathrm{BA} \rightarrow \mathrm{BS}$. It is important for us that the restriction of $\mathbf{S}$ to the category of unital Boolean algebras is an enriched contravariant Hom-set functor, because given a unital Boolean algebra $B$, the points of the space $B^{*}$ are all unital Boolean algebra morphisms $B \rightarrow \mathbf{2}$.

Stone duality for unital Boolean algebras [34] (see also the textbooks [6, 14]) states that the above described contravariant Hom-set functors to $\{0,1\}$ establish a dual equivalence between the categories of unital Boolean algebras and compact Boolean spaces. Therefore, $\{0,1\}$ is called a dualizing object, and this duality is induced by $a$ dualizing object.

Stone duality for Boolean algebras $[9,34]$ is an extension of the above duality. It states that the functors $\mathbf{A}: \mathrm{BS} \rightarrow \mathrm{BA}$ and $\mathbf{S}: \mathrm{BA} \rightarrow \mathrm{BS}$ establish a dual equivalence between the categories $B S$ and $B A$.

In this paper, we will work at the locally compact and nonunital level of generality. We will construct functors, whose restrictions to appropriate compactunital subcategories are enriched Hom-set functors, just as in the commutative case that was outlined in this subsection.

2.2. Skew Boolean algebras. A skew lattice is an algebra $(S ; \wedge, \vee)$ of type $(2,2)$ such that the operations $\wedge$ and $\vee$ are associative, idempotent and satisfy the absorption identities $x \wedge(x \vee y)=x=x \vee(x \wedge y)$ and $(y \vee x) \wedge x=x=(y \wedge x) \vee x$. The natural partial order $\leq$ on a skew lattice $S$ is defined by $x \leq y$ if and only if $x \wedge y=y \wedge x=x$ or, equivalently, $x \vee y=y \vee x=y$. A skew lattice $S$ is symmetric if $x \wedge y=y \wedge x$ which happens if and only if $x \vee y=y \vee x$. An element 0 of $S$ is called a zero 
if $x \wedge 0=0 \wedge x=0$ for all $x \in S$. The skew lattice $S$ is Boolean if it is symmetric, has a zero element and each principal order ideal $\lceil x\rceil=\{y \in S: y \leq x\}=x \wedge S \wedge x$ forms a Boolean lattice.

Let $S$ be a Boolean skew lattice and $x, y \in S$. The relative complement $x \backslash y$ is the complement of $x \wedge y \wedge x$ in the Boolean lattice $\lceil x\rceil$. A skew Boolean algebra is a Boolean skew lattice, whose signature is enriched by the nullary operation 0 and the binary relative complement operation, that is, it is an algebra $(S ; \wedge, \vee, \backslash, 0)$. Skew Boolean algebras satisfy distributivity laws $x \wedge(y \vee z)=(x \wedge y) \vee(x \wedge z)$ and $(y \vee z) \wedge x=(y \wedge x) \vee(z \wedge x)$, see [25, Theorem 2.5].

Let $S$ be a skew lattice. It is called rectangular if there exist two sets $L$ and $R$ such that $S=L \times R$, and the operations $\wedge$ and $\vee$ are defined by $(a, b) \wedge(c, d)=(a, d)$ and $(a, b) \vee(c, d)=(c, b)$. Let $\mathcal{D}$ be the equivalence relation on $S$ given by $x \mathcal{D} y$ if and only if $x \wedge y \wedge x=x$ and $y \wedge x \wedge y=y$. It is known [23, Theorem 1.7] that $\mathcal{D}$ is a congruence relation, the $\mathcal{D}$-classes of $S$ are maximal rectangular subalgebras and the quotient $S / \mathcal{D}$ is the maximal lattice image of $S$. If $S$ is a skew Boolean algebra then $S / \mathcal{D}$ is the maximal Boolean algebra image of $S$.

A skew lattice is called left-handed (right-handed) if it satisfies the identities $x \wedge y \wedge x=x \wedge y$ and $x \vee y \vee x=y \vee x$ (respectively, $x \wedge y \wedge x=y \wedge x$ and $x \vee y \vee x=$ $x \vee y$ ). In a left-handed skew Boolean algebra the rectangular subalgebras are flat in the sense that $x \mathcal{D} y$ if and only if $x \wedge y=x$ and $y \wedge x=y$. If $S$ is a left-handed skew Boolean algebra then the band $(S, \wedge)$ is left normal. It can be easily shown (or see [17]) that for each $a \in S$ and $D \in S / \mathcal{D}$ such that $[a]_{\mathcal{D}} \geq D$ there is a unique $b \in S$ such that $[b]_{\mathcal{D}}=D$ and $a \geq b$. This element $b$ is called the restriction of $a$ to $D$ and is denoted by $\left.a\right|_{D}$.

A skew Boolean algebra $S$ is called primitive if it has only one nonzero $\mathcal{D}$-class or, equivalently, if $S / \mathcal{D}$ is the Boolean algebra 2. Up to isomorphism, finite primitive lefthanded skew Boolean algebras are the algebras $\mathbf{n}+\mathbf{2}, n \geq 0$. These algebras play an important role in this paper. The underlying set of $\mathbf{n}+\mathbf{2}$ is the set $\{0,1, \ldots, n+1\}$ and its nonzero $\mathcal{D}$-class is the class $D=\{1, \ldots, n+1\}$. The operations on $D$ are determined by left-handedness: $x \wedge y=x$ and $x \vee y=y$ for any $x, y \in D$.

A skew Boolean algebra has finite intersections if any finite set of its elements has the greatest lower bound, called the intersection, with respect to the natural partial order. Since all intersections that we consider are finite, we will sometimes just write 'intersections' for 'finite intersections'. Homomorphisms of skew Boolean algebras with finite intersections are required to preserve the finite intersections.

For a detailed introduction to the theory of skew Boolean algebras we refer the reader to $[4,24,26]$.

Let $\varphi: S_{1} \rightarrow S_{2}$ be a morphism of skew Boolean algebras. We denote by $\bar{\varphi}$ : $S_{1} / \mathcal{D} \rightarrow S_{2} / \mathcal{D}$ the underlying morphism of Boolean algebras. We call $\varphi$ proper if $\bar{\varphi}$ is proper.

We fix the notation Skew for the category of left-handed skew Boolean algebras and proper morphisms. All skew Boolean algebras considered in the following are left-handed, so we take a convention to write 'skew Boolean algebra' for 'left-handed 
skew Boolean algebra'. By a morphism of skew Boolean algebras we will mean a morphism in the category Skew.

2.3. Étale spaces. Preliminaries on étale spaces can be found in any textbook on sheaf theory, for example, in [5, 29]. An étale space over $X$ is a triple $(E, f, X)$, where $E, X$ are topological spaces and $f: E \rightarrow X$ is a surjective local homeomorphism. The points of $E$ are called germs. A local section or just a section in $E$ is an open subset $A$ of $E$ such that the restriction of the map $f$ to $A$ is injective. If $U$ is an open set in $X$ then $E(U)$ is the set of all sections of $E$ over $U$, where a section $A$ is over $U$ provided that $f(A)=U$. For $x \in X$ we denote the set of all $y \in E$ such that $f(y)=x$ by $E_{x}$. This set is called the stalk over $x$. If $A \in E(U)$ then for $x \in U$ by $A(x)$ we denote the germ $y \in A \cap E_{x}$.

Let $(\mathcal{A}, g, X)$ and $(\mathcal{B}, h, Y)$ be étale spaces and $f: X \rightarrow Y$ be a continuous map. A cohomomorphism over $f$ (or an $f$-cohomomorphism) $k: \mathcal{B} \leadsto \mathcal{A}$ is a collection of maps $k_{x}: \mathcal{B}_{f(x)} \rightarrow \mathcal{A}_{x}$ for each $x \in X$ such that for every section $s \in \mathcal{B}(U)$ the function $x \mapsto k_{x}(s(f(x)))$ is a section of $\mathcal{A}$ over $f^{-1}(U)$. The maps $k_{x}$ are called the components of $k$.

We introduce the notation Etale for the category of étale spaces over Boolean spaces and their cohomomorphisms over continuous proper maps. All étale spaces considered in the following are over Boolean spaces, therefore we take a convention to write 'étale space' for 'étale space over a Boolean space'. By a morphism of étale spaces we will mean a morphism in the category Etale.

\subsection{Equivalence of the categories of skew Boolean algebras and étale spaces.}

The constructions of this paper rely on the generalization of Stone duality to lefthanded skew Boolean algebras given in [16]. We outline it in this subsection.

Let $(E, \pi, X)$ be an étale space. Denote by $E^{*}$ the set of all compact-open sections of $E$. We define binary operations $\underline{\cup}$ and $\bar{\cap}$ on $E^{*}$ as follows. Let $A, B \in E^{*}$. We set

$$
\begin{gathered}
(A \cup \underline{B})(x)= \begin{cases}B(x) & \text { if } x \in \pi(B), \\
A(x) & \text { if } x \in \pi(A) \backslash \pi(B),\end{cases} \\
(A \bar{\cap} B)(x)=A(x) \text { for all } x \in \pi(A) \cap \pi(B) .
\end{gathered}
$$

Then $A \cup B$ is a section over $\pi(A) \cup \pi(B), A \bar{\cap} B$ is a section over $\pi(A) \cap \pi(B)$ and $\left(E^{*}, \underline{\cup}, \overline{\bar{\cap}}\right)$ is a left-handed Boolean skew lattice. The associated skew Boolean algebra $E^{*}$ is called the dual skew Boolean algebra to the étale space $(E, \pi, X)$. Note that $a \mathcal{D} b$ in $E^{*}$ if and only if $\pi(a)=\pi(b)$. The assignment $[a]_{\mathcal{D}} \mapsto \pi(a)$ establishes an isomorphism between $E^{*} / \mathcal{D}$ and $X^{*}$. Note that $E^{*}$ has finite intersections if and only if $E$ is Hausdorff.

Before providing the construction in the opposite direction, we recall the definition of an ultrafilter of a poset. Let $P$ be a poset. A nonempty and strict subset $F$ of $P$ is called a filter, provided that:

(i) if $a \in F$ and $b \geq a$ then $b \in F$ ( $F$ is upwardly closed);

(ii) for any $a, b \in F$ there is $c \in F$ such that $c \leq a, b$ ( $F$ is down directed). 
A maximal filter is called an ultrafilter. In [16] we use the term prime filter for ultrafilters (note that in Boolean algebras both notions coincide). Also, we use a different approach to define ultrafilters of skew Boolean algebras. It is easy to show that the definition of ultrafilters from [16] is equivalent to the one stated above.

Let $S$ be a skew Boolean algebra. The points of the dual étale space $S^{*}$ are the ultrafilters of $S$. The latter can be characterized as follows. Let $F$ be an ultrafilter of the Boolean algebra $S / \mathcal{D}$ and let $a \in S$ be such that $[a]_{\mathcal{D}} \in F$. Then the set

$$
X_{a, F}=\left\{b \in S: \text { there is } c \in S \text { such that } a, b \geq c \text { and }[c]_{\mathcal{D}} \in F\right\}
$$

is an ultrafilter of $S$, and any ultrafilter of $S$ is of this form.

The set $S^{*}$ of all ultrafilters of $S$ is called the spectrum of $S$. There is the projection map $\hat{\pi}: S^{*} \rightarrow(S / \mathcal{D})^{*}$ that is given by $X_{a, F} \mapsto F$. For $a \in S$ we put $M(a)=\left\{F \in S^{*}: a \in\right.$ $F\}$.

The topology on $S^{*}$ is given by a subbase, that is in fact a base, consisting of the sets $M(a), a \in S$. We have that $S^{*}=\left(S^{*}, \hat{\pi},(S / \mathcal{D})^{*}\right)$ is an étale space called the dual étale space of $S$. The space $S^{*}$ is Hausdorff if and only if the skew Boolean algebra $S$ has finite intersections.

We now describe the correspondence for morphisms. Let $(E, f, X)$ and $(G, g, Y)$ be étale spaces and $k: E \rightarrow F$ a morphism. We can readily extend $k$ to the map on sections. It takes compact-open sections to compact-open sections and preserves the operations $\underline{\cup}, \bar{\cap}$ and $\varnothing$. This gives rise to the morphism $\widetilde{k}: E^{*} \rightarrow G^{*}$ of skew Boolean algebras. Note that if the spaces $E$ and $G$ are Hausdorff and all components of $k$ are injective then $\widetilde{k}$ preserves finite intersections.

Conversely, let $S, T$ be skew Boolean algebras and $k: S \rightarrow T$ a morphism. The map $\bar{k}^{-1}$ induces a morphism of Boolean spaces from $(T / \mathcal{D})^{*}$ to $(S / \mathcal{D})^{*}$. Let $F \in(T / \mathcal{D})^{*}$ and $V \in T_{F}^{*}$. Then the set $k^{-1}(V)$ is either empty or a disjoint union of several ultrafilters over $\bar{k}^{-1}(F)$. This allows to define the component $\widetilde{k}_{F}$ of the morphism $\widetilde{k}: S^{*} \rightarrow T^{*}$. The domain of $\widetilde{k}_{F}$ is $S_{\bar{k}^{-1}(F)}^{*}$ and for $U \in S_{\bar{k}^{-1}(F)}^{*}$ we set $\widetilde{k}_{F}(U)=V$ if $V \in T_{F}^{*}$ and $U \subseteq k^{-1}(V)$. Note that if $S, T$ have finite intersections and $k$ preserves them, then all components of $\widetilde{k}$ are injective.

We now state the theorem that provides insights needed for establishing the main results of this paper.

THeorem 2.1 [16]. The described assignments are functors that establish an equivalence between the categories Etale and Skew. If $S$ is a skew Boolean algebra then $S$ is naturally isomorphic to $S^{* *}$ via the map $\beta_{S}$ given by $\beta_{S}(a)=M(a), a \in S$. If $E$ is an étale space over a Boolean space then it is naturally isomorphic to $E^{* *}$ via the map $\gamma_{E}$ given by $\gamma_{E}(A)=N_{A}=\left\{N \in E^{*}: A \in N\right\}, A \in E$.

Consider an application of this theorem. Let $n \geq 0$ and $\varphi: S \rightarrow \mathbf{n}+\mathbf{2}$ be a morphism of skew Boolean algebras (such morphisms will play an important role in Section 4). We interpret this topologically. Observe that $(\mathbf{n}+\mathbf{2}) / \mathcal{D} \simeq \mathbf{2}$ and $\mathbf{2}^{*}$ is a 
one-element space, $\{a\}$. The cohomomorphism $\widetilde{\varphi}$ has therefore only one component $\widetilde{\varphi}_{a}: S_{\bar{\varphi}^{-1}(a)}^{*} \rightarrow\{1, \ldots, n+1\}$. Conversely, any map $\phi: S_{\bar{\varphi}^{-1}(a)}^{*} \rightarrow\{1, \ldots, n+1\}$ gives rise to a morphism from $S$ to $\mathbf{n}+\mathbf{2}$ over $\bar{\varphi}^{-1}$.

A noncommutative generalization of Stone duality to skew Boolean algebras with intersections is given by the following theorem.

Theorem $2.2[2,16]$. The category of left-handed skew Boolean algebras with intersections is equivalent to the category of Hausdorff étale spaces over Boolean spaces whose morphisms are cohomomorphisms with injective components.

Let $S, T$ be left-handed skew Boolean algebras with intersections. Theorems 2.1 and 2.2 tell us that, unless $S$ is commutative, the set of all morphisms from $S$ to $T$ is much bigger than the set of intersection-preserving such morphisms. For example, if $S=\mathbf{4}$ and $T=\mathbf{3}$, morphisms from $S$ to $T$ are given by functions from $\{1,2,3\}$ to $\{1,2\}$. Intersection-preserving morphisms are given by injective such functions, which means that there are no intersection-preserving morphisms at all (note that we do not take into account the zero map since we consider only proper morphisms). For the constructions of this paper, it is crucial that we consider all cohomomorphisms between appropriate spaces, and not only the ones with all components injective. So it is important that the duality theorem we use in this paper is Theorem 2.1 and not Theorem 2.2.

We now explain why the unital versions of the dualities of Theorems 2.1 and 2.2 are not induced by a dualizing object. We emphasize that this explanation is applicable only to the particular kinds of morphisms given in Theorems 2.1 and 2.2. For Theorem 2.1, consider the functor constructing the spectrum of a skew Boolean algebra. As follows from [16, Lemma 6.3], points of the spectrum of a skew Boolean algebra $S$ are in a bijective correspondence with morphisms $S \rightarrow \mathbf{n}+\mathbf{2}$ such that the inverse image of 1 is nonempty and minimal possible. This does not produce all morphisms $S \rightarrow \mathbf{n}+\mathbf{2}$ (and neither all morphisms from $S$ to another skew Boolean algebra), even in the case when $S / \mathcal{D}$ is unital. For the example from the previous paragraph, there are exactly $2^{3}=8$ morphisms from 4 to 3 . But only three of these morphisms, that are listed below, give rise to the points of the spectrum of 4 :

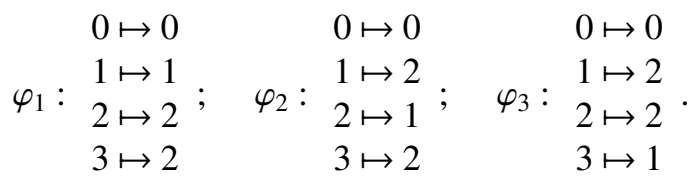

For Theorem 2.2, this follows from the fact that for any skew Boolean algebra with intersections $T$, there exists another skew Boolean algebra with intersections $S$ such that there are no intersection-preserving morphisms from $S$ to $T$ at all (we have only to take care that any stalk of $S^{*}$ has cardinality sticktly greater than the cardinality of any stalk of $T^{*}$ ). 


\section{The functors $\lambda_{n}, n \geq 0$}

Let $X$ be a Boolean space and let $n \geq 0$ be fixed. We regard the set $\{0, \ldots, n+1\}$ as a discrete topological space. Let $\lambda_{n}(X)$ denote the set of all continuous maps $f$ from $X$ to $\{0, \ldots, n+1\}$ such that the sets $f^{-1}(1), \ldots, f^{-1}(n+1)$ are compact. Define the binary operations $\wedge$ and $\vee$ on $\lambda_{n}(X)$ to be induced by the operations $\wedge$ and $\vee$ on the primitive left-handed skew Boolean algebra $\mathbf{n}+\mathbf{2}$. That is, for $f, g \in \lambda_{n}(X)$ we put

$$
(f \wedge g)(x)=f(x) \wedge g(x), \quad(f \vee g)(x)=f(x) \vee g(x) .
$$

With respect to $\wedge$ and $\vee$ the set $\lambda_{n}(X)$ becomes a left-handed Boolean skew lattice. By adding to its signature the relative complement operation and the zero, we turn it into a left-handed skew Boolean algebra. Note that $\lambda_{0}(X)=X^{*}$.

It is immediate that the elements of $\lambda_{n}(X)$ are in a bijective correspondence with ordered $(n+1)$-tuples of pairwise disjoint compact-open subsets of $S$ via the assignment $f \mapsto\left(f^{-1}(1), \ldots, f^{-1}(n+1)\right)$.

REMARK 3.1. Another realization of $\lambda_{n}(X)$ is by flags $A_{n+1} \supseteq \cdots \supseteq A_{1}$ of compact-open subsets of $X$ via the assignment

$$
f \mapsto f^{-1}(\{1, \ldots, n+1\}) \supseteq f^{-1}(\{1, \ldots, n\}) \supseteq f^{-1}(1) .
$$

Let $A_{n+1} \supseteq \cdots \supseteq A_{1}$ and $B_{n+1} \supseteq \cdots \supseteq B_{1}$ be the flags corresponding to $f$ and $g$, respectively. It is easy to verify, applying the definitions of $\vee$ and $\wedge$, that the flag corresponding to $f \vee g$ is $C_{n+1} \supseteq \cdots \supseteq C_{1}$, where $C_{i}=\left(A_{i} \backslash B_{n+1}\right) \cup B_{i}$ for all $i$, and the flag corresponding to $f \wedge g$ is $D_{n+1} \supseteq \cdots \supseteq D_{1}$, where $D_{i}=A_{i} \cap B_{n+1}$ for all $i$. From this description it follows that $\lambda_{1}(X)=\omega(\mathbf{A}(X))$, where $\mathbf{A}$ is the functor from Subsection 2.1 and $\omega: \mathrm{BA} \rightarrow$ Skew is the (version with proper morphisms of the) Leech-Spinks $\omega$-functor [27]. Therefore, the construction of $\lambda_{1}$ provides a new interpretation of the functor $\omega$.

Let us look at the structure of the skew Boolean algebra $\lambda_{n}(X)$ in more detail. For $f \in \lambda_{n}(X)$ by $\hat{f}: X \rightarrow\{0,1\}$ we denote the map given by

$$
\hat{f}^{-1}(1)=f^{-1}(\{1, \ldots, n+1\}) .
$$

Lemma 3.2. Let $f, g \in \lambda_{n}(X)$. Then $f \mathcal{D} g$ if and only if

$$
f^{-1}(\{1, \ldots, n+1\})=g^{-1}(\{1, \ldots, n+1\}) .
$$

The quotient Boolean algebra $\lambda_{n}(X) / \mathcal{D}$ is isomorphic to $\lambda_{0}(X)=X^{*}$ via the map $[f]_{\mathcal{D}} \mapsto \hat{f}$.

Proof. By definition, $f \mathcal{D} g$ is equivalent to $f \wedge g=f$ and $g \wedge f=g$. Therefore, $f(x) \wedge g(x)=f(x)$ and $g(x) \wedge f(x)=g(x)$ for all $x \in X$. The latter equalities are equivalent to the condition $f(x)=0$ if and only if $g(x)=0$ for all $x \in X$. This is equivalent to $f^{-1}(\{1, \ldots, n+1\})=g^{-1}(\{1, \ldots, n+1\})$. The second statement is easy to verify. 
Lemma 3.3. The natural partial order on $\lambda_{n}(X)$ is given by $f \geq g$ if and only if

$$
f^{-1}(i) \supseteq g^{-1}(i) \quad \text { for all } i \in\{1, \ldots, n+1\} \text {. }
$$

Proof. By definition, $f \geq g$ is equivalent to $f \wedge g=g \wedge f=g$. By the definition of the operation $\wedge$ on $\lambda_{n}(X)$, the latter is equivalent to

$$
f(x) \wedge g(x)=g(x) \wedge f(x)=g(x)
$$

for all $x \in X$, where the equalities are in $\mathbf{n}+\mathbf{2}$. This is equivalent to the condition that $g(x)=i$ implies $f(x)=i$ for all $i \in\{1, \ldots, n+1\}$.

Lemma 3.4. The skew Boolean algebra $\lambda_{n}(X)$ has finite intersections. If $f, g \in \lambda_{n}(X)$ then the intersection $f \cap g$ is given by

$$
(f \cap g)^{-1}(i)=f^{-1}(i) \cap g^{-1}(i)
$$

for all $i \in\{1, \ldots, n+1\}$.

Proof. Let $h \in \lambda_{n}(X)$ be given by $h^{-1}(i)=f^{-1}(i) \cap g^{-1}(i)$ for all $i \in\{1, \ldots, n+1\}$. Then $f, g \geq h$ by Lemma 3.3. Assume that $f, g \geq q$ and let $i \in\{1, \ldots, n+1\}$. Then $f^{-1}(i) \supseteq q^{-1}(i)$ and $g^{-1}(i) \supseteq q^{-1}(i)$. It follows that $h^{-1}(i) \supseteq q^{-1}(i)$. This proves that $f \cap g$ exists and equals $h$.

It is well known that the ultrafilters of the Boolean algebra $\lambda_{0}(X)$ are the sets $\left\{f \in \lambda_{0}(X): f(x)=1\right\}$. Consequently, the ultrafilters of the Boolean algebra $\lambda_{n}(X) / \mathcal{D}$ are the sets

$$
N_{x}=\left\{[f]_{\mathcal{D}} \in \lambda_{n}(X) / \mathcal{D}: f(x) \in\{1, \ldots, n+1\}\right\}, \quad x \in X .
$$

Let $\alpha: \lambda_{n}(X) \rightarrow \lambda_{n}(X) / \mathcal{D}$ be the projection map.

Lemma 3.5. For every $x \in X$ and $i \in\{1, \ldots, n+1\}$ the set

$$
N_{x, i}=\left\{f \in \lambda_{n}(X): f(x)=i\right\}
$$

is an ultrafilter of $\lambda_{n}(X)$, and any ultrafilter is of this form. We also have $\alpha\left(N_{x, i}\right)=N_{x}$. Proof. Let $f \in N_{x, i}$. We show that $N_{x, i}=X_{f, N_{x}}$. Let $g \in N_{x, i}$. Since $x \in f^{-1}(i) \cap g^{-1}(i)$ we have $f \cap g \in N_{x, i}$. Thus also $[f \cap g]_{\mathcal{D}} \in N_{x}$. This and $f, g \geq f \cap g$ imply that $g \in X_{f, N_{x}}$.

Conversly, let $g \in X_{f, N_{x}}$. Then there is some $h \in \lambda_{n}(X)$ with $[h]_{\mathcal{D}} \in N_{x}$ such that $f, g \geq h$. Since $f \geq h$ and $h(x) \neq 0$ it follows that $h(x)=i$ by Lemma 3.3. Now, $g \geq h$ implies $g(x)=i$ again by Lemma 3.3. It follows that $g \in N_{x, i}$, as required. The second claim is immediate.

Corollary 3.6. For any ultrafilter $N_{x}$ of $\lambda_{n}(X) / \mathcal{D}$

$$
\alpha^{-1}\left(N_{x}\right)=\bigcup_{i=1}^{n+1} N_{x, i} .
$$

Consequently, each stalk of the étale space $\left(\lambda_{n}(X)\right)^{*}$ has cardinality $n+1$. 
Proof. Ultrafilters of $\lambda_{n}(X)$ that are over $N_{x}$ are of the form $X_{f, N_{x}}$, where $f(x) \in$ $\{1, \ldots, n+1\}$. The statement now follows from the equality $N_{x, i}=X_{f, N_{x}}$, where $f(x)=i$, established in the proof of Lemma 3.5.

Let $X^{(i)}=\left\{N_{x, i}\right\}_{x \in X}, i \in\{1, \ldots, n+1\}$. It follows from Lemma 3.5 that, as a set, $\left(\lambda_{n}(X)\right)^{*}$ is the union $X^{(1)} \cup \cdots \cup X^{(n+1)}$ of $(n+1)$ disjoint copies of $X$.

Lemma 3.7. The space $\left(\lambda_{n}(X)\right)^{*}$ as a topological space is a disjoint union of $(n+1)$ copies of the Boolean space $X$.

Proof. Consider each $X^{(i)}$ as a topological space homeomorphic to $X$ via the map $N_{x, i} \mapsto x$. Then $\left(\lambda_{n}(X)\right)^{*}$ can be considered as a disjoint union of the spaces $X^{(i)}$. We aim to show that this topology coincides with the dual étale space topology on $\left(\lambda_{n}(X)\right)^{*}$, whose construction was outlined in Subsection 2.4. It is enough to verify that the map $\pi:\left(\lambda_{n}(X)\right)^{*} \rightarrow X$ given by $N_{x, i} \mapsto x$ is a local homeomorphism. Fix some $N_{x, i}$ and a compact-open set $A$ in $X$ such that $x \in A$. Consider the function $f \in \lambda_{n}(X)$ given by $f^{-1}(i)=A$ and $f^{-1}(j)=\varnothing, j \in\{1, \ldots, n+1\} \backslash\{i\}$. It is clear that $M(f)=\bigcup_{y \in A} N_{y, i}$. This is a neighborhood of $N_{x, i}$ that is homeomorphic via $\pi$ to $A$ since any basic open subset that is contained in $M(f)$ is equal to $\bigcup_{y \in B} N_{y, i}$ for some compact-open subset $B$ of $A$.

We now define $\lambda_{n}$ on morphisms. Let $n \geq 0$ and $g: X_{1} \rightarrow X_{2}$ be a morphism of Boolean spaces. For any $f \in \lambda_{n}\left(X_{2}\right)$ we put

$$
\lambda_{n}(g)(f)=f g .
$$

It is easy to check that $\lambda_{n}(g)$ is a skew Boolean algebra morphism from $\lambda_{n}\left(X_{2}\right)$ to $\lambda_{n}\left(X_{1}\right)$ and that this makes $\lambda_{n}$ a contravariant functor from the category BS to the category Skew.

We finish this section by recording the following fact that will be needed in Section 5 .

Lemma 3.8. Let $g: X_{1} \rightarrow X_{2}$ be a morphism of Boolean spaces. Then

$$
{\overline{\lambda_{n}(g)}}^{-1}\left(N_{x}\right)=N_{g(x)}
$$

and

$$
\lambda_{n}(g)^{-1}\left(N_{x, i}\right)=N_{g(x), i}
$$

for any $x \in X_{1}$ and any $i \in\{1, \ldots, n+1\}$.

Proof. We have $\overline{\lambda_{n}(g)}\left([f]_{\mathcal{D}}\right)=[f g]_{\mathcal{D}}$. Therefore, $\overline{\lambda_{n}(g)}\left([f]_{\mathcal{D}}\right) \in N_{x}$ if and only if $[f g]_{\mathcal{D}} \in N_{x}$. By (3.1) this is equivalent to $f g(x) \in\{1, \ldots, n+1\}$. Therefore, $[f]_{\mathcal{D}} \in$ $N_{f(x)}$, which implies the first equality. For the second equality, observe that

$$
\lambda_{n}(g)(f) \in N_{x, i} \Leftrightarrow f g(x) \in N_{x, i} \Leftrightarrow f \in N_{g(x), i} .
$$




\section{The functors $\Lambda_{n}$ and the adjunctions $\Lambda_{n}+\lambda_{n}, n \geq 0$}

Let $S$ be a skew Boolean algebra, $\alpha: S \rightarrow S / \mathcal{D}$ the canonical projection and $\hat{\alpha}$ the projection from $S^{*}$ to $(S / \mathcal{D})^{*}$ given by $\hat{\alpha}\left(X_{a, F}\right)=F$. We also fix $n \geq 0$.

Let $\{0,1, \ldots, n+1\}^{S}$ be the set of all maps from $S$ to $\{0,1, \ldots, n+1\}$. We regard $\{0,1, \ldots, n+1\}$ as a discrete space and $\{0,1, \ldots, n+1\}^{S}$ as a product space. That is, a base of the topology on $\{0,1, \ldots, n+1\}^{S}$ is formed by the sets

$$
U_{\delta}=\bigcap_{t \in T}\left\{f \in\{0,1, \ldots, n+1\}^{S}: f(t)=\delta(t)\right\},
$$

where $T$ runs through the finite subsets of $S$ and $\delta$ runs through the functions from $T$ into $\{0,1, \ldots, n+1\}$.

We denote by $\Lambda_{n}(S)$ the set of all morphisms from $S$ to $\mathbf{n}+\mathbf{2}$ in the category Skew. We endow $\Lambda_{n}(S)$ with the subspace topology inherited from the product topology on the space $\{0,1, \ldots, n+1\}^{S}$.

For each $s \in S$ and $i \in\{0,1, \ldots, n+1\}$ we put

$$
L(s, i)=\left\{f \in \Lambda_{n}(S): f(s)=i\right\} .
$$

Lemma 4.1. The sets $L(s, i)$, where $s$ runs through $S$ and $i$ runs through the set $\{1, \ldots, n+1\}$, form a subbase of the topology on $\Lambda_{n}(S)$.

Proof. By the definition of subspace topology and in view of the base given by (4.1), the sets

$$
V_{\delta}=U_{\delta} \cap \Lambda_{n}(S)=\bigcap_{t \in T}\left\{f \in \Lambda_{n}(S): f(t)=\delta(t)\right\},
$$

where $T$ runs through the finite subsets of $S$ and $\delta$ runs through the functions from $T$ into $\{0,1, \ldots, n+1\}$, form a base of the topology on $\Lambda_{n}(S)$. Therefore, the topology on $\Lambda_{n}(S)$ admits a subbase consisting of the sets $L(s, i), s \in S, i \in\{0,1, \ldots, n+1\}$. Let $s \in S$. We show that the set $L(s, 0)$ can be expressed as a union of some sets $L(t, i)$ such that $t \in S$ and $i \in\{1, \ldots, n+1\}$. Let

$$
A=\left\{t \in \Lambda_{n}(S): \alpha(t) \wedge \alpha(s)=0\right\} .
$$

We aim to show that

$$
L(s, 0)=\bigcup_{t \in A} \bigcup_{i=1}^{n+1} L(t, i) .
$$

Let $f \in L(s, 0)$. Since $f$ is nonzero, there is $t \in S$ such that $f(t) \neq 0$. Observe that $f\left(\left.s\right|_{\alpha(s) \wedge \alpha(t)}\right)=0$, since $s \geq\left. s\right|_{\alpha(s) \wedge \alpha(t)}$ and $f(s)=0$. It follows that $f\left(\left.t\right|_{\alpha(s) \wedge \alpha(t)}\right)=0$ as well since the two elements $\left.s\right|_{\alpha(s) \wedge \alpha(t)}$ and $\left.t\right|_{\alpha(s) \wedge \alpha(t)}$ are in the same $\mathcal{D}$-class. Hence $f\left(\left.t\right|_{\alpha(t) \backslash \alpha(s)}\right) \neq 0$. We obtain $\left.t\right|_{\alpha(t) \backslash \alpha(s)} \in A$ and $f \in \bigcup_{i=1}^{n+1} L\left(\left.t\right|_{\alpha(t) \backslash \alpha(s)}, i\right)$, proving that $L(s, 0) \subseteq \bigcup_{t \in A} \bigcup_{i=1}^{n+1} L(t, i)$.

To prove the reverse inclusion, we let $f \in \bigcup_{t \in A} \bigcup_{i=1}^{n+1} L(t, i)$. We have $\alpha(s \wedge t)=$ $\alpha(s) \wedge \alpha(t)=0$, so that $s \wedge t=0$. It follows that $f(s \wedge t)=f(0)=0$. On the other hand, we have $f(s \wedge t)=f(s) \wedge f(t)$. We obtain $0=f(s) \wedge f(t)$ and $f(t) \neq 0$. It follows that $f(s)=0$ and thus $f \in L(s, 0)$. This finishes the proof of (4.2). 
Lemma 4.2. Let $s \in S$ and $i \in\{1, \ldots, n+1\}$. The set $L(s, i)$ is a closed subset of the space $\{0, \ldots, n+1\}^{S}$. Consequently, $L(s, i)$ is a compact-open subset of $\Lambda_{n}(S)$.

Proof. Let $Y_{\wedge}$ and $Y_{\vee}$ denote the sets of all functions from $S$ to $\{0,1, \ldots, n+1\}$ that preserve $\wedge$ or $\vee$, respectively. Further, let $Y_{0}$ and $Y_{s, i}$ denote the sets of all functions from $S$ to $\{0,1, \ldots, n+1\}$ that map 0 to 0 or that map $s$ to $i$, respectively. It can be shown using a standard argument (see, for example, [14, proof of Lemma 1, Ch. 34, page 326]) that each of the sets $Y_{\wedge}, Y_{\vee}, Y_{0}$ and $Y_{s, i}$ is a closed subset of $\{0,1, \ldots$, $n+1\}^{S}$. It follows that $L(s, i)$ is closed, too, since $L(s, i)=Y_{\wedge} \cap Y_{\vee} \cap Y_{0} \cap Y_{s, i}$.

For the second claim, observe that $L(s, i)$ is compact in $\{0, \ldots, n+1\}^{S}$, because it is a closed subset of a compact space. Therefore, $L(s, i)$ is compact-open in $\Lambda_{n}(S)$.

Theorem 4.3. $\Lambda_{n}(S)$ is a Boolean space.

Proof. $\Lambda_{n}(S)$ is Hausdorff as a subspace of the Hausdoeff space $\{1, \ldots, n+1\}^{S}$. So, in view of Lemmas 4.1 and $4.2, \Lambda_{n}(S)$ is a Hausdorff space, in which compact-open sets form a base of the topology. Thus $\Lambda_{n}(S)$ is a Boolean space.

We now define $\Lambda_{n}$ on morphisms. Let $h: S_{1} \rightarrow S_{2}$ be a morphism of skew Boolean algebras. For each $f \in \Lambda_{n}\left(S_{2}\right)$ we set

$$
\left(\Lambda_{n}(h)\right)(f)=f h
$$

Lemma 4.4. $\Lambda_{n}(h)$ is a morphism of Boolean spaces from $\Lambda_{n}\left(S_{2}\right)$ to $\Lambda_{n}\left(S_{1}\right)$.

Proof. It is immediate that for each $f \in \Lambda_{n}\left(S_{2}\right)$

$$
\left(\Lambda_{n}(h)\right)(f) \in \Lambda_{n}\left(S_{1}\right)
$$

To show that $\Lambda_{n}(h)$ is a continuous proper map, it is enough to verify that the set $\left(\Lambda_{n}(h)\right)^{-1}(L(s, i))$ is compact-open for any $s \in S_{2}$ and any $i \in\{1, \ldots, n+1\}$. We observe that

$$
\begin{aligned}
f \in\left(\Lambda_{n}(h)\right)^{-1}(L(s, i)) & \Longleftrightarrow\left(\Lambda_{n}(h)\right)(f) \in L(s, i) \\
& \Longleftrightarrow f h \in L(s, i) \Longleftrightarrow f(h(s))=i \Longleftrightarrow f \in L(h(s), i),
\end{aligned}
$$

implying that $\left(\Lambda_{n}(h)\right)^{-1}(L(s, i))=L(h(s), i)$, and the statement follows.

It is straightforward to check that the constructed assignments define a contravariant functor $\Lambda_{n}$ : Skew $\rightarrow$ BS.

We are now prepared to formulate and prove our adjunction theorem.

THEOREM 4.5. For each $n \geq 0$ the functor $\Lambda_{n}$ : Skew $\rightarrow$ BS $^{o p}$ is the left adjoint to the functor $\lambda_{n}: \mathrm{BS}^{\mathrm{op}} \rightarrow$ Skew. For a skew Boolean algebra $S$ the component $\eta_{S}$ of the unit of the adjunction $\eta$ is given by

$$
\eta_{S}(a)(g)=g(a), \quad a \in S, g \in \Lambda_{n}(S) .
$$


Proof. It is immediate that $\eta_{S}$ is a morphism. Let $S$ be a skew Boolean algebra, $X$ a Boolean space and $\mu: S \rightarrow \lambda_{n}(X)$ a morphism of skew Boolean algebras. Our aim is to show that there is a unique morphism $u: X \rightarrow \Lambda_{n}(S)$ of Boolean spaces such that $\mu=\lambda_{n}(u) \eta_{S}$.

For each $x \in X$ we put

$$
u(x)(s)=\mu(s)(x), \quad s \in S
$$

Let us verify that $u$ is a proper continuous map. For this, we have to show that the inverse image under $u$ of a compact-open subset of $\Lambda_{n}(S)$ is compact-open in $X$. Since any compact-open subset is a finite union of basic compact-open sets, and any basic compact-open set is a finite intersection of the sets of the form $L(s, i)$, it is enough to verify that $u^{-1}(L(s, i))$ is compact-open in $X$ for each $s \in S$ and each $i \in\{1, \ldots, n+1\}$.

$$
\begin{aligned}
x \in u^{-1}(L(s, i)) & \Leftrightarrow u(x) \in L(s, i) \Leftrightarrow u(x)(s)=i \\
& \Leftrightarrow \mu(s)(x)=i \Leftrightarrow x \in \mu(s)^{-1}(i) .
\end{aligned}
$$

Since $\mu(s) \in \lambda_{n}(X)$, we have that $\mu(s)^{-1}(i)$ is compact-open by the definition of $\lambda_{n}(X)$. So $u^{-1}(L(s, i))$ is compact-open, too.

We verify the equality $\mu=\lambda_{n}(u) \eta_{S}$. For each $a \in S$ and $x \in X$

$$
\begin{aligned}
\left(\lambda_{n}(u) \eta_{S}(a)\right)(x) & =\eta_{S}(a) u(x) \quad(\text { by }(3.2)) \\
& =u(x)(a) \quad(\text { by }(4.3)) \\
& =\mu(a)(x) \quad(\text { by }(4.4))
\end{aligned}
$$

as required.

The uniqueness of $u$ can be shown in a standard way.

Remark 4.6. Let $S$ be a skew Boolean algebra and $\mathbf{A}, \mathbf{S}$ the functors from Subsection 2.1. We set $\Omega=\mathbf{A} \Lambda_{1}$ : Skew $\rightarrow$ BA. It follows from Theorem 4.5 that $\Omega$ is the left adjoint to the functor $\omega=\lambda_{1} \mathbf{S}: \mathrm{BA} \rightarrow$ Skew from [27]. The construction of $\Lambda_{1}$ yields the full description of (the version with proper morphisms of) $\Omega$. This extends the result of [27], where the action of $\Omega$ on finite objects was described.

The statement that follows provides a way to faithfully represent a skew Boolean algebra $S$ as a subalgebra of a well-understood skew Boolean algebra with intersections $\lambda_{n} \Lambda_{n}(S)$. Each stalk of its dual étale space has cardinality $n+1$. If $n=1$, we obtain a faithful representation of $S$ in a skew Boolean algebra with intersections $\lambda_{1} \Lambda_{1}(S)$. Each stalk of its dual space has cardinality 2. This algebra has, roughly speaking, a 'low degree of noncommutativity' (but note that its underlying Boolean algebra $\left(\Lambda_{1}(S)\right)^{*}$ is rather huge in comparison with the underlying Boolean algebra $S / \mathcal{D}$ of $S)$. In the terminology of Leech and Spinks [27], $\lambda_{1} \Lambda_{1}(S)$ is a minimal skew Boolean cover of the Boolean algebra $\left(\Lambda_{1}(S)\right)^{*}$. We refer the reader to [27] for a detailed discussion of this notion. 
THeOREM 4.7. For each $n \geq 1$ the map $\eta_{S}$ is injective, and is therefore a faithful representation of $S$ as a subalgebra of $\lambda_{n} \Lambda_{n}(S)$.

Proof. Let $a, b \in S$ and $a \neq b$. Then $M(a) \neq M(b)$ by Theorem 2.1. Thus we can assume that there is an ultrafilter $X_{a, F}$ in $S$ such that $b \notin X_{a, F}$ (but note that $a \in X_{a, F}$ ). Let $x$ be the only point of the space $\mathbf{2}^{*}$. The map $g: \mathbf{2}^{*} \rightarrow(S / \mathcal{D})^{*}$ given by $x \mapsto F$ is a continuous map. As follows from the discussion in the paragraph after Theorem 2.1, we can construct a cohomomorphism $k: S^{*} \rightarrow(\mathbf{n}+\mathbf{2})^{*}$ over $g$ given by $k_{x}\left(X_{a, F}\right)=1$ and $k_{x}(G)=2$ for any $G \in S_{F}^{*}$ such that $G \neq X_{a, F}$. Since $b \notin X_{a, F}$ we have $k(b) \neq 1$. Therefore, the morphism from $S$ to $\mathbf{n}+\mathbf{2}$ that corresponds to $k$ has different values at $a$ and $b$. This implies that $\eta_{S}(a)(k) \neq \eta_{S}(b)(k)$.

\section{The algebras of the monads of the adjunctions $\Lambda_{n}+\lambda_{n}$}

Preliminaries on monads can be found in, for example, [1, Ch. 10] or [28, Ch. VI]. Let $n \geq 0$ be fixed throughout this section.

Let $(T, \eta, \mu)$ be the monad over the category Skew that arises from the adjunction $\Lambda_{n} \dashv \lambda_{n}$. We have $T=\lambda_{n} \Lambda_{n}, \eta$ is the unit of the adjunction given by (4.3), and $\mu=\lambda_{n} \epsilon_{\Lambda_{n}}$ is a natural transformation from $T^{2}$ to $T$, where $\epsilon: 1_{\mathrm{BS}} \rightarrow \Lambda_{n} \lambda_{n}$ is the counit of the adjunction.

The following statement is straightforward to verify.

Lemma 5.1. Let $X$ be a Boolean space. Then

$$
\epsilon_{X}(x)(f)=f(x), \quad x \in X, f \in \lambda_{n}(X) .
$$

Let $S$ be a skew Boolean algebra. In this section we will work with skew Boolean algebras $\lambda_{n} \Lambda_{n} \lambda_{n} \Lambda_{n}(S), \lambda_{n} \Lambda_{n}(S)$ and morphisms between them. These objects are rather complicated, and therefore we first find a convenient way of working with them. We start from a useful description of points of the space $\Lambda_{n}(S)$.

Lemma 5.2. There is a bijective correspondence between the points of the space $\Lambda_{n}(S)$ and elements of the set

$$
\left\{(F, f): F \in(S / \mathcal{D})^{*}, f \in\{1, \ldots, n+1\}^{S_{F}^{*}}\right\} .
$$

Proof. Let $a$ be the only point of the Boolean space $((\mathbf{n}+\mathbf{2}) / \mathcal{D})^{*} \simeq \mathbf{2}^{*}$. Let $h \in \Lambda_{n}(S)$. Then $\bar{h}^{-1}$ induces a continuous proper map, that we denote also by $\bar{h}^{-1}$, from $2^{*}$ to $(S / \mathcal{D})^{*}$. Let $F_{h} \in(S / \mathcal{D})^{*}$ be such that $\bar{h}^{-1}(a)=F_{h}$. Then $\widetilde{h}$ has only one component $\widetilde{h}_{a}: S_{F_{h}}^{*} \rightarrow(\mathbf{n}+\mathbf{2})_{a}^{*}=\{1, \ldots, n+1\}$. We show that

$$
h \mapsto\left(F_{h}, \widetilde{h}_{a}\right)
$$

is a bijection. Assume that $\left(F_{h}, \widetilde{h}_{a}\right)=\left(F_{g}, \widetilde{g}_{a}\right)$. Then we have $\bar{h}=\bar{g}$ and $h^{-1}(i)=g^{-1}(i)$ for all $i \in\{1, \ldots, n+1\}$. Therefore, (5.2) is injective. For the reverse direction, let $F \in(S / \mathcal{D})^{*}$ and $f \in\{1, \ldots, n+1\}^{S_{F}^{*}}$. The map $g$ from $2^{*}$ to $(S / \mathcal{D})^{*}$ given by $g(a)=F$ is proper and continuous. Hence $f$ is the only component of a $g$-cohomomorphism from $S^{*}$ to $(\mathbf{n}+\mathbf{2})^{*}$. This and Theorem 2.1 imply that $(5.2)$ is surjective. 
We now characterize the points of the space $\Lambda_{n} \lambda_{n} \Lambda_{n}(S)$. Let $\mathcal{T}_{n+1}$ denote the set of all transformations of the set $\{1, \ldots, n+1\}$.

Lemma 5.3. There is a bijective correspondence between the points of the space $\Lambda_{n} \lambda_{n} \Lambda_{n}(S)$ and elements of the set

$$
\left\{(F, f, g): F \in(S / \mathcal{D})^{*}, f \in\{1, \ldots, n+1\}^{S_{F}^{*}}, g \in \mathcal{T}_{n+1}\right\} .
$$

Proof. Let $h \in \Lambda_{n} \lambda_{n} \Lambda_{n}(S)$, that is, $h$ is a skew Boolean algebra morphism from $\lambda_{n} \Lambda_{n}(S)$ to $\mathbf{n}+2$. Then $\bar{h}$ is a Boolean algebra morphism from $\left(\lambda_{n} \Lambda_{n}(S)\right) / \mathcal{D}$ to 2 . Since $\bar{h}^{-1}(1)$ is an ultrafilter of $\left(\lambda_{n} \Lambda_{n}(S)\right) / \mathcal{D}$, it is equal to some $N_{G}$, where $G \in$ $\Lambda_{n}(S)$, see (3.1). The only component of the cohomomorphism $\tilde{h}$ is a map from $\left(\lambda_{n} \Lambda_{n}(S)\right)_{N_{G}}^{*}$ to $\{1, \ldots, n+1\}$. This map defines $g \in \mathcal{T}_{n+1}$, where $N_{G, i} \mapsto g(i)$ for all $i \in\{1, \ldots, n+1\}$. By Lemma 5.2, $G$ corresponds to some pair $(F, f)$. Hence $h$ corresponds to the triple $(F, f, g)$. Finally, similarly as in the proof of Lemma 5.2, it can be shown that this correspondence is bijective.

To proceed, we need to describe the action of the maps that are involved in the construction of the monad $(T, \eta, \mu)$, provided that the points of the spaces $\Lambda_{n}(S)$ and $\Lambda_{n} \lambda_{n} \Lambda_{n}(S)$ are identified with the elements of the sets (5.1) and (5.3) via the constructions in the proofs of Lemmas 5.2 and 5.3, respectively. We do this in the following three lemmas. By id we denote the identity transformation of the set $\{1, \ldots, n+1\}$.

Lemma 5.4. $\epsilon_{\Lambda_{n}(S)}(F, f)=(F, f, \mathrm{id})$.

Proof. Let $\varphi \in \Lambda_{n}(S)$ and assume that $\epsilon_{\Lambda_{n}(S)}(\varphi)=\varphi^{\prime}$. By Lemma 5.1 we have $\varphi^{\prime}(g)=$ $g(\varphi)$ for each $g \in \lambda_{n} \Lambda_{n}(S)$. Thus for all $i$

$$
g \in \varphi^{\prime-1}(i) \Leftrightarrow \varphi \in g^{-1}(i) \Leftrightarrow g \in N_{\varphi, i}
$$

Therefore, $\varphi^{\prime-1}(i)=N_{\varphi, i}$. Thus also ${\overline{\varphi^{\prime}}}^{-1}(1)=N_{\varphi}$. This and the constructions in the proofs of Lemmas 5.2 and 5.3 imply that if $\varphi$ corresponds to $(F, f)$ then $\varphi^{\prime}$ corresponds to $(F, f, \mathrm{id})$, as required.

LeMma 5.5. ${\overline{\eta_{S}}}^{-1}\left(N_{(F, f)}\right)=F$ and the component ${\widetilde{\eta_{S}}}_{N_{(F, f)}}$ of $\widetilde{\eta_{S}}$ is given by

$$
x \mapsto N_{(F, f), f(x)}, \quad x \in S_{F}^{*} .
$$

Proof. Let $\varphi \in \Lambda_{n}(S)$. For any $a \in S$ and any $i \in\{1, \ldots, n+1\}$, applying the definition of $N_{\varphi, i}$ and (4.3), we write

$$
a \in \eta_{S}^{-1}\left(N_{\varphi, i}\right) \Leftrightarrow \eta_{S}(a) \in N_{\varphi, i} \Leftrightarrow \eta_{S}(a)(\varphi)=i \Leftrightarrow \varphi(a)=i \Leftrightarrow a \in \varphi^{-1}(i) .
$$

Thus $\eta_{S}^{-1}\left(N_{\varphi, i}\right)=\varphi^{-1}(i)$. If $\varphi$ corresponds to the pair $(F, f)$ via the construction in the proof of Lemma 5.2, then the only component of $\tilde{\varphi}$ is $f: S_{F}^{*} \rightarrow\{1, \ldots, n+1\}$. We therefore have ${\widetilde{\eta_{S}}}^{-1}\left(N_{(F, f), i}\right)=f^{-1}(i)$, and the statement follows. 
LeMma 5.6. ${\overline{\mu_{S}}}^{-1}=\epsilon_{\Lambda_{n}(S)}^{* *}$, that is, ${\overline{\mu_{S}}}^{-1}\left(N_{x}\right)=N_{\epsilon_{\Lambda_{n}(S)}(x)}$ and the component ${\widetilde{\mu_{S}}}_{N_{(F, f)}}$ is given by

$$
N_{(F, f, \text { id }), i} \mapsto N_{(F, f), i}
$$

Proof. Note that $\mu_{S}=\lambda_{n}\left(\epsilon_{\Lambda_{n}(S)}\right)$ and apply Lemmas 3.8 and 5.4.

We now characterize the algebras for the monad $(T, \eta, \mu)$. An algebra of the monad $(T, \eta, \mu)$ (or just a $T$-algebra) is a pair $(S, \gamma)$ such that $S$ is a skew Boolean algebra and $\gamma: T(S) \rightarrow S$ is a morphism such that the following diagrams commute:
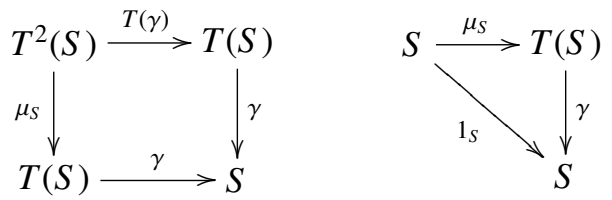

A morphism of T-algebras $h:\left(S_{1}, \gamma\right) \rightarrow\left(S_{2}, \delta\right)$ is a morphism $h: S_{1} \rightarrow S_{2}$ such that the following diagram commutes:

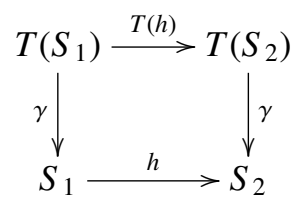

Theorem 5.7. (1) A pair $(S, \gamma)$ is an algebra for the monad $(T, \eta, \mu)$ if and only if $S=\lambda_{n}(X)$ for some Boolean space $X$ and $\gamma=\lambda_{n} \epsilon_{X}$.

(2) A map $h: \lambda_{n}\left(X_{1}\right) \rightarrow \lambda_{n}\left(X_{2}\right)$ is a morphism of $T$-algebras if and only if $h=\lambda_{n}(f)$ for some morphism $f: X_{1} \rightarrow X_{2}$.

Proof. The equality $1_{S}=\gamma \eta_{S}$ implies the equality $1_{(S / D)^{*}}=\bar{\eta}_{S}-1 \bar{\gamma}^{-1}$. Applying Theorem 2.1 we have the following commuting diagrams:
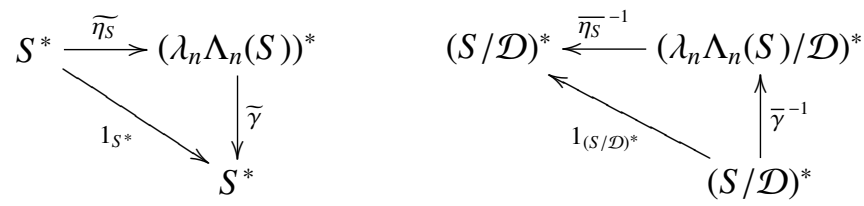

Let $F \in(S / \mathcal{D})^{*}$. Assume that $\bar{\gamma}^{-1}(F)=N_{(G, f)}$. By Lemma 5.5 we have $\bar{\eta}_{S}^{-1}\left(N_{(G, f)}\right)=G$. Since the second diagram above commutes, $F=G$. We fix $f=$ $f_{F}: S_{F}^{*} \rightarrow\{1, \ldots, n+1\}$ such that $\bar{\gamma}^{-1}(F)=N_{(F, f)}$. Since the first diagram above commutes and applying Lemma 5.5, we see that for any $x \in S_{F}^{*}$

$$
x \stackrel{{\widetilde{\eta_{S}}}_{N_{(F, f)}}}{\longrightarrow} N_{(F, f), f(x)} \stackrel{\widetilde{\gamma_{F}}}{\longrightarrow} x .
$$


The latter shows that both the map ${\widetilde{\eta_{S}}}_{N_{(F, f)}}$ and the restriction of the map $\widetilde{\gamma_{F}}$ to the image of ${\widetilde{\eta_{S}}}_{N_{(F, f)}}$ are injective. Injectivity of ${\widetilde{\eta_{S}}}_{N_{(F, f)}}$ implies that $f$ has to be injective, too.

By definition and applying Theorem 2.1 we have the following diagrams (where $\left.\Lambda_{n}(\gamma)^{* *}\left(N_{x}\right)=N_{\Lambda_{n}(\gamma)(x)}\right)$ :
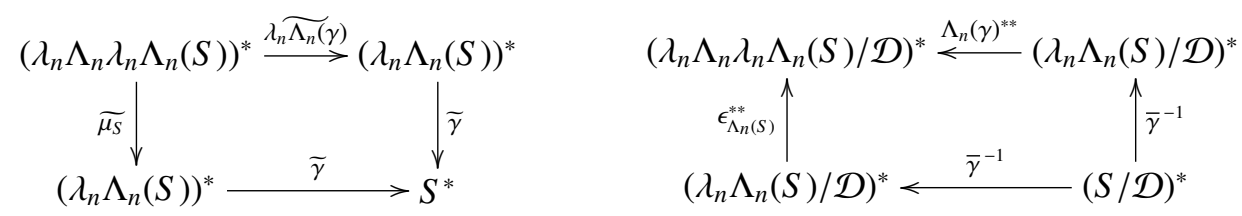

Our goal now is to describe the action of $\Lambda_{n}(\gamma)$ and $\lambda_{n} \Lambda_{n}(\gamma)$ provided that the elements of the spaces are encoded by the elements of the sets (5.1) and (5.3). Let $g \in \Lambda_{n}(S)$. It corresponds to the pair $(F, \hat{g})$, where $F=\bar{g}^{-1}(1)$ and $\hat{g}$ is the only component of $\widetilde{g}$. Applying $\Lambda_{n}(\gamma)(g)=g \gamma$ and the construction in the proof of Lemma 5.3, we can write

$$
(F, \hat{g}) \stackrel{\Lambda_{n}(\gamma)}{\longrightarrow}\left(F, f, \hat{g} \widetilde{\gamma}_{F}\right)
$$

This and Lemma 3.8 yield

$$
N_{\left(F, f, \hat{g} \widetilde{\gamma_{F}}\right), i} \stackrel{\lambda_{n} \widetilde{\Lambda_{n}}(\gamma)_{(F, \hat{g})}^{\longrightarrow}}{\longrightarrow} N_{(F, \hat{g}), i}
$$

for each $i \in\{1, \ldots, n+1\}$. It follows that for any $F \in(S / \mathcal{D})^{*}$ we can write

$$
F \stackrel{\bar{\gamma}^{-1}}{\longrightarrow} N_{(F, f)} \stackrel{\Lambda_{n}(\gamma)^{* *}}{\longrightarrow} N_{\left(F, f, f \widetilde{\gamma}_{F}\right)}
$$

On the other hand, observe that $\left(\lambda_{n} \Lambda_{n}(S) / \mathcal{D}\right)^{*}$ is isomorphic to $\Lambda_{n}(S)$ and $\left(\lambda_{n} \Lambda_{n} \lambda_{n} \Lambda_{n}(S) / \mathcal{D}\right)^{*}$ is isomorphic to $\Lambda_{n} \lambda_{n} \Lambda_{n}(S)$ via the map $N_{x} \mapsto x$. Applying Lemma 5.4, for any $F \in(S / \mathcal{D})^{*}$

$$
F \stackrel{\bar{\gamma}^{-1}}{\longrightarrow} N_{(F, f)} \stackrel{\epsilon_{\Lambda_{n}(S)}^{* *}}{\longrightarrow} N_{(F, f, \text { id })}
$$

Since (5.4) and (5.5) are equal, $f$ is a bijection and $\widetilde{\gamma}_{F}=f^{-1}$. Hence $\left|S_{F}^{*}\right|=n+1$. Therefore, each stalk of $S^{*}$ has cardinality $n+1$.

Let $F \in(S / \mathcal{D})^{*}$ be fixed. Since $\left|S_{F}^{*}\right|=n+1$ and $f$ is a bijection, we can enumerate the germs of $S_{F}^{*}$ so that $S_{F}^{*}=\left\{F_{(1)}, \ldots, F_{(n+1)}\right\}$ and $f$ maps each $F_{(i)}$ to $i$. In this notation

$$
\widetilde{\gamma}_{F}\left(N_{(F, f), i}\right)=F_{(i)} \text {. }
$$

Let $i \in\{1, \ldots, n+1\}$. We define the ith layer of the space $S^{*}$ as the set

$$
S_{(i)}^{*}=\left\{F_{(i)}: F \in(S / \mathcal{D})^{*}\right\} .
$$

We show that $S \simeq \lambda_{n}\left((S / \mathcal{D})^{*}\right)$. It is convenient to work with the isomorphic copy $S^{* *}$ of $S$. Let $s \in S$. Observe that $\widetilde{\eta_{S}}\left(M(s) \cap S_{(i)}^{*}\right)$ is the ith layer of $M\left(\eta_{S}(s)\right)$. Since 
the projection of the latter set is compact-open, its inverse image under $\bar{\gamma}$ is compactopen, too. It follows that the map $F_{(i)} \mapsto N_{F, i}$, where $F \in(S / \mathcal{D})^{*}$ and $i \in\{1, \ldots, n+1\}$, induces an isomorphism between $S^{* *}$ and $\lambda_{n}\left((S / \mathcal{D})^{*}\right)^{* *}$.

Let $X=(S / \mathcal{D})^{*}$. To establish the equality $\gamma=\lambda_{n} \epsilon_{X}$, we apply arguments similar to those in the proofs of Lemmas 5.4 and 5.6 to observe that the action of $\widetilde{\lambda_{n} \epsilon_{X F}}$ coincides with the action of $\widetilde{\gamma}_{F}$ given in (5.6).

We are left to prove the claim about morphisms. Let $f: X_{2} \rightarrow X_{1}$ be a morphism of Boolean spaces. It is straightforward to verify that

$$
\lambda_{n}(f): \lambda_{n}\left(X_{1}\right) \rightarrow \lambda_{n}\left(X_{2}\right)
$$

is a morphism of $T$-algebras from $\left(T\left(\lambda_{n}\left(X_{1}\right)\right), \lambda_{n} \epsilon_{X_{1}}\right)$ to $\left(T\left(\lambda_{n}\left(X_{2}\right)\right), \lambda_{n} \epsilon_{X_{2}}\right)$.

We now assume that $h: \lambda_{n}\left(X_{1}\right) \rightarrow \lambda_{n}\left(X_{2}\right)$ is a morphism of $T$-algebras from $\left(T\left(\lambda_{n}\left(X_{1}\right)\right), \lambda_{n} \epsilon_{X_{1}}\right)$ to $\left(T\left(\lambda_{n}\left(X_{2}\right)\right), \lambda_{n} \epsilon_{X_{2}}\right)$. Since $\left(\lambda_{n}\left(X_{i}\right) / \mathcal{D}\right)^{*} \simeq X_{i}, i=1,2$, we have that $\bar{h}^{-1}$ induces a morphism, $\hat{h}$, from $X_{2}$ to $X_{1}$. We show that $h=\lambda_{n}(\hat{h})$. Let $(F, f) \in \Lambda_{n}\left(S_{2}\right)$, where $F \in X_{2}$ and $f \in\{1, \ldots, n+1\}^{\left(\lambda_{n}\left(X_{2}\right)\right)_{F}^{*}}$. Then

$$
(F, f) \stackrel{\Lambda_{n}(h)}{\longrightarrow}\left(\bar{h}^{-1}(F), \tilde{f h_{F}}\right),
$$

and therefore

$$
N_{\left(\bar{h}^{-1}(F), \tilde{\left.h_{F}\right), i}\right.} \stackrel{\lambda_{n} \widetilde{\Lambda_{n}}(h)_{N_{(F, f)}}}{\longrightarrow} N_{(F, f), i}
$$

for all $i \in\{1, \ldots, n+1\}$.

Let $X$ be a Boolean space. Similarly as is done in Lemma 5.3, we may establish a bijection between the points of $\Lambda_{n} \lambda_{n}(X)$ and the pairs $(F, f), F \in X, f \in \mathcal{T}_{n+1}$. If $g \in \Lambda_{n} \lambda_{n}(X)$ then $\bar{g}: X^{*} \rightarrow \mathbf{2}$ determines $F$ and the only component of $\widetilde{g}$ determines $f$. By Lemma 3.8

$$
N_{(F, \text { id }), i} \stackrel{\widetilde{\lambda_{n} \epsilon_{X_{2}}}}{\longrightarrow} N_{(F, i)}
$$

From (5.7), (5.8) and the commutative diagram

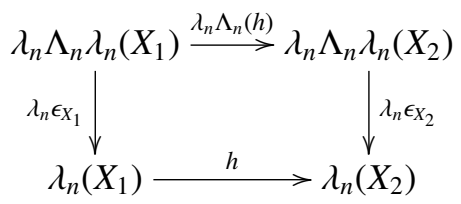

we see that $\widetilde{\lambda_{n} \epsilon_{X_{1}}}$ is defined on all $N_{\left(\bar{h}^{-1}(F), \widetilde{h}_{F}\right), i}, F \in X_{2}, i \in\{1, \ldots, n+1\}$, and moreover $\widetilde{h}_{F}=\mathrm{id}$. Hence the action of $\widetilde{h}_{F}$ is given by $N_{\bar{h}^{-1}(F), i} \mapsto N_{F, i}$ which implies that $h=\lambda_{n}(\hat{h})$.

Let $\lambda_{n}(\mathrm{BS})$ be the category whose objects are $\lambda_{n}(X)$, where $X$ is a Boolean space, and whose arrows are $\lambda_{n}(f)$, where $f$ is a morphism of Boolean spaces. 
Corollary 5.8. The category of Eilenberg-Moore algebras of the monad $(T, \eta, \mu)$ is isomorphic to the category $\lambda_{n}(\mathrm{BS})$. Consequently, the adjunction $\Lambda_{n} \dashv \lambda_{n}$ is monadic for every $n \geq 0$.

Proof. The first statement follows from Theorem 5.7. The second statement holds because the category $\lambda_{n}(\mathrm{BS})$ is obviously isomorphic to the category $\mathrm{BS}^{\mathrm{op}}$.

COROLlaRY 5.9. The category $\lambda_{n}(\mathrm{BS})$ is a reflective subcategory of the category Skew. The reflector is given by the functor $\Lambda_{n} \lambda_{n}$.

\section{Acknowledgements}

I would like to thank the anonymous referee, in particular, for suggesting that the topology on the space $\Lambda_{n}(S)$ might coincide with the subspace topology of the product space $\{0, \ldots, n+1\}^{S}$. This turned out to be the case and led to a significant simplification of the proof that $\Lambda_{n}(S)$ is a Boolean space in Section 4. I am also grateful to Marcel Jackson and Mark Lawson for helpful comments.

\section{References}

[1] S. Awodey, Category Theory (Oxford University Press, New York, 2006).

[2] A. Bauer and K. Cvetko-Vah, 'Stone duality for skew Boolean algebras with intersections', Houston J. Math. 39(1) (2013), 73-109.

[3] A. Bauer, K. Cvetko-Vah, M. Gehrke, S. van Gool and G. Kudryavtseva, 'A noncommutative Priestley duality'. arXiv: 1206.5848.

[4] R. J. Bignall and J. E. Leech, 'Skew Boolean algebras and discriminator varieties', Algebra Universalis 33 (1995), 387-398.

[5] G. E. Bredon, Sheaf Theory, Graduate Texts in Mathematics, 170 (Springer-Verlag, New York, 1997).

[6] S. Burris and H. P. Sankappanavar, A Course in Universal Algebra, Graduate Texts in Mathematics, 78 (Springer-Verlag, New York-Berlin, 1981).

[7] D. M. Clark and B. A. Davey, Natural Dualities for the Working Algebraist (Cambridge University Press, Cambridge, 1998).

[8] W. H. Cornish, 'Boolean skew algebras', Acta Math. Acad. Sci. Hungar. 36 (1980), 281-291.

[9] H. P. Doctor, 'The categories of Boolean lattices, Boolean rings and Boolean spaces', Canad. Math. Bull. 7 (1964), 245-252.

[10] J. Funk, 'Semigroups and toposes', Semigroup Forum 75(3) (2007), 481-520.

[11] J. Funk and P. Hofstra, 'Topos theoretic aspects of semigroup actions', Theory Appl. Categ. 24(6) (2010), 117-147.

[12] J. Funk, M. V. Lawson and B. Steinberg, 'Characterizations of Morita equivalent inverse semigroups', J. Pure Appl. Algebra 215(9) (2011), 2262-2279.

[13] J. Funk and B. Steinberg, 'The universal covering of an inverse semigroup', Appl. Categ. Structures 18(2) (2010), 135-163.

[14] S. Givant and P. Halmos, Introduction to Boolean Algebras, Undergraduate Texts in Mathematics (Springer, New York, 2009).

[15] P. T. Johnstone, Stone Spaces (Cambridge University Press, Cambridge, 1986).

[16] G. Kudryavtseva, 'A refinement of Stone duality to skew Boolean algebras', Algebra Universalis 67 (2012), 397-416.

[17] G. Kudryavtseva and M. V. Lawson, 'The structure of generalized inverse semigroups', Semigroup Forum, to appear; arXiv:1207.4296. 
[18] G. Kudryavtseva and M. V. Lawson, 'The classifying space of an inverse semigroup'. arXiv: 1210.4421.

[19] G. Kudryavtseva and M. V. Lawson, 'Stone duality for generalized inverse semigroups', in preparation.

[20] M. V. Lawson, 'A noncommutative generalization of Stone duality', J. Aust. Math. Soc. 88 (2010), 385-404.

[21] M. V. Lawson, 'Non-commutative Stone duality: inverse semigroups, topological groupoids and $C^{\star}$-algebras', Internat. J. Algebra Comput. 22(6) (2012).

[22] M. V. Lawson and D. H. Lenz, 'Pseudogroups and their étale groupoids', Adv. Math. 244 (2013), $117-170$.

[23] J. Leech, 'Skew lattices in rings', Algebra Universalis 26 (1989), 48-72.

[24] J. Leech, 'Skew Boolean Algebras', Algebra Universalis 27 (1990), 497-506.

[25] J. Leech, 'Normal Skew lattices', Semigroup Forum 44 (1992), 1-8.

[26] J. Leech, 'Recent developments in the theory of skew lattices', Semigroup Forum 52 (1996), 7-24.

[27] J. Leech and M. Spinks, 'Skew Boolean algebras derived from Boolean algebras', Algebra Universalis 58(3) (2008), 287-302.

[28] S. Mac Lane, Categories for the Working Mathematician, Graduate Texts in Mathematics, 5 (Springer-Verlag, New York, 1998).

[29] S. Mac Lane and I. Moerdijk, 'Sheaves in geometry and logic', in: A First Introduction to Topos Theory (Springer, New York, 1994).

[30] H.-E. Porst and W. Tholen, 'Concrete dualities', in: Category Theory at Work (Heldermann Verlag, Berlin, 1991), 111-136.

[31] P. Resende, 'Étale groupoids and their quantales', Adv. Math. 208 (2007), 147-209.

[32] P. Resende, 'Lectures on étale groupoids, inverse semigroups and quantales', Preprint available at www.math.ist.utl.pt.

[33] B. Steinberg, 'Strong Morita equivalence of inverse semigroups', Houston J. Math. 37 (2011), 895-927.

[34] M. H. Stone, 'Applications of the theory of Boolean rings to general topology', Trans. Amer. Math. Soc. 41 (1937), 375-481.

GANNA KUDRYAVTSEVA, Faculty of Computer and Information Science, University of Ljubljana, Tržaška cesta, 25, SI-1001, Ljubljana, Slovenia e-mail: ganna.kudryavtseva@fri.uni-lj.si 